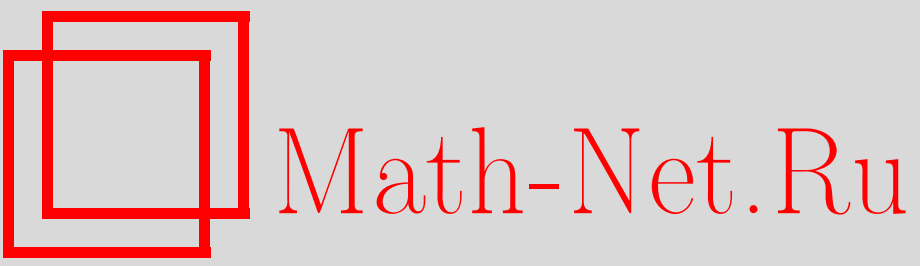

А. А. Станиславский, Вероятностная интерпретация интеграла дробного порядка, TMФ, 2004, том 138, номер 3, 491-507

DOI: https://doi.org/10.4213/tmf34

Использование Общероссийского математического портала Math-Net.Ru подразумевает, что вы прочитали и согласны с пользовательским соглашением

http://www.mathnet.ru/rus/agreement

Параметры загрузки:

IP: 34.229 .45 .116

26 апреля 2023 г., 04:46:55 
ТЕОРЕТИЧЕСКАЯ

И МАТЕМАТИЧЕСКАЯ

ФИЗИКА

Том 138, № 3

март, 2004

(C) 2004 г.

А. А. Станиславский*

\title{
ВЕРОЯТНОСТНАЯ ИНТЕРПРЕТАЦИЯ ИНТЕГРАЛА ДРОБНОГО ПОРЯДКА
}

\begin{abstract}
Установлена связь между устойчивыми распределениями теории вероятности и дробным интегралом. При этом оказывается, что параметр устойчивого распределения совпадает с показателем дробного интеграла. Из анализа полученных результатов следует, что уравнения с дробной производной по времени описывают эволюцию некоторой физической системы, у которой временная степень свободы становится стохастической, т.е. она представляет собой сумму случайных временных отрезков, подчиняющихся устойчивому вероятностному распределению. Обсуждается связь между фрактальным множеством Кантора (полосками Кантора) и дробным интегралом. Показано, что эта связь имеет весьма ограниченное применение в качестве аппроксимации дробного интеграла.
\end{abstract}

Ключевые слова: интеграл дробного порядка, устойчивые вероятностные распределения, уравнение Фоккера-Планка, множество Кантора, субдиффузия, сверхмедленная релаксация.

\section{1. ВВЕДЕНИЕ}

Интегральное и дифференциальное исчисления дробного порядка начались с работ Абеля, Римана, Лиувилля и развивались параллельно с традиционными исчислениями целого порядка, фактически их обобщая. На протяжении XIX и XX столетий этот раздел математики был хорошо разработан и исследован [1], [2]. Сейчас он широко применяется для описания, например, “сверхмедленной” релаксации в диэлектриках и ферроэлектриках [3], [4], процессов диффузии [5]-[7], гамильтонова хаоса [8], [9]. Но если исчисление производных и интегралов целого порядка имеет ясную геометрическую и физическую интерпретации, то для дробных интегрирования и дифференцирования такая интерпретация отсутствует. Несомненно, ее установление существенно упростило бы решение и понимание многих фундаментальных и прикладных научных задач. Поэтому неудивительно, что этот вопрос был включен в список открытых проблем на первой международной конференции, посвяшенной интегральному и дифференциальному исчислению дробного порядка в Нью Хавен (США, 1974) [10]. Затем он неоднократно обсуждался на последующих конференциях в Глазго (Великобритания, 1984) [11],

*Радиоастрономический институт НАН Украины, Харьков, Украина. E-mail: alexstan@ira.kharkov.ua 
Токио (Япония, 1989) [12], Варне (Болгария, 1996) [13]. Тем не менее до настоящего времени приемлемого решения этой проблемы не найдено.

В связи с проникновением идей фрактальной геометрии [14] в теоретическую физику [15] предпринимались активные попытки показать связь между фрактальным множеством Кантора и дробным интегралом [16]-[18]. Исходной посылкой этого подхода является причинный интеграл типа свертки

$$
f(t)=\int_{0}^{t} g(t-\tau) h(\tau) d \tau,
$$

который преобразует входной сигнал $h(t)$ в выходной сигнал $f(t)$ с помошью функции памяти (импульсного отклика) $g(t)$. Если

$$
g(t)=\left\{\begin{array}{l}
1 \text { для } t \geqslant 0, \\
0 \text { для } t<0
\end{array}\right.
$$

- ступенчатая функция, то выражение (1) будет простым интегралом первого порядка. А если $g(t)=\delta(t)-\delta$-функция Дирака, то преобразование (1) воспроизводит входной сигнал (интегрирование нулевого порядка). Логично предположить, что дробное интегрирование порядка $\nu(0<\nu<1)$

$$
f(t)=\frac{1}{\Gamma(\nu)} \int_{0}^{t}(t-\tau)^{\nu-1} h(\tau) d \tau
$$

будет интерполировать функцию памяти между $\delta$-функцией (полное отсутствие памяти) и ступенчатой функцией (полная память). В работе [16] предложено осушествить эту интерполяцию с помощью полосок Кантора на временном интервале $[0, t]$ следуюшим образом. Сначала удаляется средняя часть этого интервала и на концах остаются два одинаковых отрезка длины $\xi t(\xi<1 / 2)$. Каждый сохранившийся отрезок длины $\xi t$ подвергается той же самой процедуре, а сама процедура рекуррентно повторяется до бесконечности. На каждом полученном временном интервале строится полоска. Относительная высота полосок выбирается такой, чтобы совокупная плошадь всех полосок на каждом этапе разбиения сохранялась постоянной. Согласно [16] утверждается, что предельный переход $N \rightarrow \infty$ ( $N$ - номер этапа построения множества Кантора) этой процедуры сходится к дробному интегралу. Математическая непоследовательность такой интерполяции была убедительно показана в работах [19].

Позже этот подход был пересмотрен и проанализирован более детально в книге [17]. Здесь множество Кантора уже строится на отрезке $[0, T]$, где $T$ превьшает длительность самого процесса. Это позволяет преодолеть предыдущее противоречие с понятием свертки. Теперь учитывается лог-периодическое поведение множества Кантора при $N \rightarrow \infty$, которое устраняется усреднением по периоду $\ln \xi$. Однако эта модификация не спасает общее положение. Как установлено в [20], такая процедура усреднения гладкой функции по множеству Кантора дает только асимптотически (с точностью до константы) ядро в свертке (1), равное произведению степенной функции 
на лог-периодическую. Поэтому она весьма грубо аппроксимирует дробный интеграл и не имеет какого-либо преимущества. Такой результат скорее говорит о том, что прямая связь между исчислением дробного порядка и фрактальной геометрией пока не установлена. Это в полной мере относится и к работам [18], которые используют результаты или идеи работ [16], [17].

В этой статье представлен новый подход к интерпретации интегрального и дифференциального исчислений дробного порядка. Основная цель заключается в том, чтобы показать связь между устойчивыми распределениями теории вероятности и дробным интегралом. В нашем рассмотрении временная степень свободы является стохастической. Она представляет собой сумму случайных временных отрезков, и каждый из них является случайной величиной устойчивого вероятностного распределения. Существует математически обоснованный предельный переход от дискретных временных “шагов" (отрезков) к непрерывному пределу. На этом пути строятся процессы с рандомизированным операционным временем. Кинетические уравнения, описывающие такие процессы, записываются через производные (или интегралы) дробного порядка по времени. Показатель дробного интеграла (производной) имеет прямую связь с параметром соответствующего устойчивого распределения вероятности. Приводится выражение функции памяти после процедуры усреднения гладкой функции по множеству Кантора. Она имеет более простой вид, чем предложенный в [17]. Обсуждаются причины независимости результатов [17] от перехода с множества Кантора, состоящего из двух полосок на каждой стадии разбиения, на произвольные и/или случайные разбиения.

\section{2. СЛУЧАЙНЫЕ БЛУЖДАНИЯ С РАНДОМИЗИРОВАННЫМ ОПЕРАЦИОННЫМ ВРЕМЕНЕМ}

Начнем анализ с дискретной модели случайных блужданий, которые имеют случайные времена ожидания $T_{i}$ между скачками случайной длины $R_{i}$. Эта модель впервые была введена Монтроллом и Вейссом [21] и нашла широкое применение для описания сильной турбулентности, хаотических систем, релаксационных явлений, транспортных процессов в случайных средах [22]. Пусть $T_{1}, T_{2}, \ldots$ будут неотрицательными независимыми идентично распределенными случайными величинами, принадлежашими устойчивому вероятностному распределению с параметром $\alpha$. Следует заметить, что индекс $\alpha$ находится в строго определенном диапазоне $0<\alpha<1$ в силу неотрицательности значений времен ожидания $T_{i}$. Такая случайная величина имеет характеристическую функцию (преобразование Фурье от функции распределения вероятности $F(x)$ ) вида

$$
f(s)=\int_{-\infty}^{\infty} \exp (i s x) d F(x)=\exp \left[i \gamma s-\sigma\left(1-i \operatorname{tg}\left(\frac{\pi \alpha}{2}\right)\right)\right]
$$

где $\sigma \geqslant 0$ и $\gamma$ - действительные параметры [23]. Тогда сумма $b_{n}\left(T_{1}+\cdots+T_{n}\right)$ с параметром нормировки $b_{n}=n^{-1 / \alpha}>0(n \in \mathbf{N}$ - множество натуральных чисел) сходится к процессу с устойчивым распределением индекса $\alpha$. Этот процесс полезно охарактеризовать параметром $\tau$ (локальное время). Пока мы рассматриваем дискретную модель, 
локальное время $\tau$ принимает дискретные значения с интервалом $\delta \tau$. Существует предельный переход от дискретных временных шагов к непрерывному пределу [24]. Обозначим через $\lfloor x\rfloor$ целую часть величины $x$. Процесс $b_{\lfloor\tau / \delta \tau\rfloor+1} \sum_{i=1}^{\lfloor\tau / \delta \tau\rfloor+1} T_{i}$ при $\delta \tau \rightarrow 0$ сходится по распределению к процессу $T(\tau) \stackrel{d}{=} \tau^{1 / \alpha} T(1)$, где $\stackrel{d}{=}$ означает равенство по распределению, а $T(1) \stackrel{d}{=} T_{1}$. Такой предельный процесс является марковским, строго $\alpha$-стабильным и асимметричным. При $\tau \rightarrow \infty$ величина $T(\tau) \rightarrow \infty$, а это значит, что любая выборочная траектория такого процесса будет неубывающей (почти наверное, почти всюду). Процесс $T(\tau)$ является самоподобным с показателем $1 / \alpha>1$ [25], т.е. $\{T(c \tau)\}_{\tau \geqslant 0} \stackrel{\text { f.d. }}{=}\left\{c^{1 / \alpha} T(\tau)\right\}_{\tau \geqslant 0}$ для всех $c>0$, где $\stackrel{\text { f.d. }}{=}$ обозначает равенство всех конечномерных распределений.

Теперь определим случайные скачки в пространстве. Пусть они имеют одинаковое гауссово распределение вероятности и будут независимыми друг от друга и от $T_{i}$. Не ограничивая обшности нашего рассмотрения, положим блуждания одномерными. Используя предельный переход от дискретных шагов к непрерывным скачкам, мы приходим к стохастическому процессу $\{R(\tau)\}_{\tau} \geqslant 0$, удовлетворяющему соотношению самоподобия $\{R(c \tau)\}_{\tau \geqslant 0} \stackrel{\text { f.d. }}{=}\left\{c^{1 / 2} R(\tau)\right\}_{\tau \geqslant 0}$ для всех $c>0$. Как и $T(\tau)$, процесс $R(\tau)$ зависит от непрерывного внутреннего параметра $\tau$, который отличается от реального времени $t$.

Чтобы определить положение частицы в реальном (истинном) времени при таком нерегулярном движении, необходимо найти число скачков частицы к моменту времени $t$. Для дискретного процесса число скачков $N_{t}=\max \left\{n \in \mathbb{N} \mid \sum_{i=1}^{n} T_{i} \leqslant t\right\}$, а положение частицы $r_{N_{t}}=\sum_{i=1}^{N_{t}} R_{i}$. Непрерывный предел $N_{t}$ приводит к процессу $S(t)=$ $\inf \{x \mid T(x)>t\}$, удовлетворяюшему соотношению $S(T(\tau))=\tau$ (почти наверное, почти всюду), а поэтому его можно считать обратным к $T(\tau)$. Поскольку процесс $T(\tau)$ строго возрастающий, то $S(t)$ будет неубывающим. Из свойства самоподобия процесса $T(\tau)$ прямо следует, что $\{S(c t)\}_{t \geqslant 0} \stackrel{\text { f.d. }}{=}\left\{c^{\alpha} S(t)\right\}_{t \geqslant 0}$ для всех $c>0$. Хотя $T(\tau)-$ процесс Леви, его обратный $S(t)$ не является процессом Леви и даже марковским процессом. Как показано в [24], он представляет собой непрерывный субмартингал. Любая выборочная траектория процесса $S(t)$ будет только возрастаюшей. Тогда положение частицы $r_{t}$ в реальный момент времени $t$ определяется подчиненным процессом $R(S(t))$. Напомним, что подчиненный процесс $Y(U(t))$ получается рандомизацией временной переменной случайного процесса $Y(t)$, использующего в таком случае новый “таймер”, случайный процесс $U(t)$ с неотрицательными независимыми приращениями. Говорят, что результируюший процесс $Y(U(t))$ является подчиненным к $Y(t)$ и направляется процессом $U(t)$, называемым направляющим. Направляющий процесс иначе назьвают рандомизированным операционным временем [26]. Подчиненный процесс $R(S(t))$ является самоподобным с индексом $\alpha / 2$, т.е. $\{R(S(c t))\}_{t \geqslant 0} \stackrel{\text { f.d. }}{=}\left\{c^{\alpha / 2} R(S(t))\right\}_{t \geqslant 0}$ для всех $c>0$. Следует отметить, что хотя сам процесс $R(\tau)$ - марковский, подчиненный процесс $R(S(t))$ может это свойство утратить (это будет показано ниже). Фактически в данном контексте радиус-вектор частищы $r_{t}$ представляет собой рандомизацию параметра $\tau$ обычного броуновского движения $\mathcal{B}_{\tau}$. 
Плотность вероятности радиус-вектора $r_{t}$ описьвается интегральным соотношением

$$
p^{r_{t}}(t, x)=\int_{0}^{\infty} p^{R}(\tau, x) p^{S}(t, \tau) d \tau
$$

где $p^{R}(\tau, x)$ - вероятность найти процесс $R(\tau)$ в точке $x$ в момент операционного времени $\tau$, a $p^{S}(t, \tau)$ - вероятность совпадения операционного времени $\tau$ и истинного времени $t$. Случайная величина $S(t)$ обладает тем важным свойством [24], что среднее

$$
\left\langle e^{-v S(t)}\right\rangle=\sum_{n=0}^{\infty} \frac{\left(-v t^{\alpha}\right)^{n}}{\Gamma(1+n \alpha)}=E_{\alpha}\left(-v t^{\alpha}\right)
$$

выражается через функцию Миттаг-Лефлера, где Г $(z)$ - гамма-функция (эйлеров интеграл второго рода). Это позволяет найти плотность вероятности $S(t)$ в явном виде $p^{S}(t, \tau)=t^{-\alpha} F_{\alpha}\left(\tau / t^{\alpha}\right)$. Необходимые вычисления и обсуждение свойств функции $F_{\alpha}(z)$ приведены в приложении.

Поскольку

$$
\int_{0}^{\infty} p^{R}(\tau, x) e^{-u^{\alpha} \tau} d \tau=\hat{p}^{R}\left(u^{\alpha}, x\right),
$$

то в пространстве Лапласа плотность вероятности $p^{r_{t}}(t, x)$ имеет простой вид: $u^{\alpha-1} \times$ $\hat{p}^{R}\left(u^{\alpha}, x\right)$. Для дальнейшего анализа полезно воспользоваться формулой

$$
p^{r_{t}}(t, x)=\int_{0}^{\infty} F_{\alpha}(z) p^{R}\left(t^{\alpha} z, x\right) d z .
$$

Эта функция неотрицательна и удовлетворяет условию нормировки

$$
\int_{-\infty}^{\infty} p^{r_{t}}(t, x) d x=\int_{0}^{\infty} F_{\alpha}(z) d z=1
$$

Граничная величина $\alpha=1$ может быть тоже добавлена к нашему рассмотрению. Это вырожденный случай, когда $T(\tau)=\tau, S(t)=t$. Плотность вероятности $p^{S}(t, \tau)$ сводится к $\delta$-функции Дирака, и после интегрирования получается плотность вероятности нормальной диффузии $p^{r}(x, t)=p^{R}(x, t)$. Результаты этого раздела легко обобшаются на произвольную размерность пространства, а сам процесс $R(\tau)$ может быть даже операторно-устойчивым [25]. Следует также отметить, что одной из возможных причин возникновения устойчивого распределения для времени ожидания $T(\tau)$ рассматриваемого здесь случайного блуждания может быть захват частицы отдельными случайными ловушками (частичная локализация). Важность этого механизма неоднократно обсуждалась в литературе [6]-[9], [18], [22]. 
ПримеР 1. Предположим, что процесс $R(\tau)$ имеет простой гауссов вид с плотностью вероятности $(\pi D \tau)^{-1 / 2} e^{-x^{2} /(D \tau)}$, где $D$ - константа. Тогда выражение $(4)$ может быть проинтегрировано до конца. С помощью преобразования Фурье функции Гаусса по переменной $x$

$$
\frac{1}{\sqrt{\pi z D t^{\alpha}}} \int_{-\infty}^{\infty} e^{-x^{2} /\left(D t^{\alpha} z\right)+i k x} d x=e^{-z k^{2} D t^{\alpha} / 4},
$$

мы вычисляем интеграл

$$
\int_{0}^{\infty} F_{\alpha}(z) e^{-z k^{2} D t^{\alpha} / 4} d z=E_{\alpha}\left(-\frac{k^{2} D t^{\alpha}}{4}\right) .
$$

Как было упомянуто выше, функция Миттаг-Леффлера $E_{\alpha}\left(-k^{2} D t^{\alpha} / 4\right)$ имеет преобразование Лапласа по переменной $t$ в виде $u^{\alpha-1} /\left(u^{\alpha}+k^{2} D / 4\right)$. Находим обратное преобразование Фурье по переменной $k$, а затем обратное преобразование Лапласа по переменной u. В результате получаем

$$
p^{r_{t}}(t, x)=D^{-1 / 2} t^{-\alpha / 2} F_{\alpha / 2}\left(\frac{2|x|}{\sqrt{D} t^{\alpha / 2}}\right) .
$$

Интересно, что в этом частном случае плотность вероятности $p^{r_{t}}(t, x)$ выражается через функцию $F_{\alpha / 2}(z)$. Все нечетные моменты для $r_{t}$ равны нулю, а четные моменты

$$
\left\langle r_{t}^{2 n}\right\rangle=\left(\frac{D t^{\alpha}}{4}\right)^{n} \int_{0}^{\infty} z^{2 n} F_{\alpha / 2}(z) d z=\frac{\left(D t^{\alpha}\right)^{n}(2 n) !}{4^{n} \Gamma(1+\alpha n)} .
$$

Следовательно, сам процесс $r_{t}$ при $0<\alpha<1$ ведет себя как субдиффузия. Этот результат был ранее получен с помошью другого подхода в работе [27]. Если положить $\alpha=1$, выражение (5) будет описывать обычное броуновское движение (нормальную диффузию). Константу $D$ можно интерпретировать как обобшенный коэффишиент диффузии с размерностью $[D]=$ длина ${ }^{2} /$ время $^{\alpha}$.

\section{3. УРАВНЕНИЕ ФОККЕРА-ПЛАНКА С ДРОБНОЙ ПРОИЗВОДНОЙ ПО ВРЕМЕНИ}

Оказьвается, индекс $\alpha$ в выражении (4) характеризует долговременные эффекты памяти подчиненного процесса $r_{t}$. В этом разделе мы обсудим этот важный факт более детально. Обозначим через $\hat{L}$ не зависящий от времени оператор Фоккера-Планка, чья точная форма не важна для дальнейшего рассмотрения. Пусть плотность вероятности $p^{R}(R, \tau)$ удовлетворяет стандартному уравнению Фоккера-Планка

$$
\frac{\partial p^{R}(R, \tau)}{\partial \tau}=\hat{L} p^{R}(R, \tau)
$$

Напомним, что преобразование Лапласа функции $p^{r_{t}}(R, t)$ по переменной $t$ имеет вид $\hat{p}^{r_{t}}(R, u)=u^{\alpha-1} \hat{p}^{R}\left(R, u^{\alpha}\right)$. Действуя оператором $\hat{L}$ на изображение Лапласа $\hat{p}^{r_{t}}(R, u)$, 
мы получаем $\left[\hat{L} \hat{p}^{r_{t}}\right](R, u)=u^{\alpha} \hat{p}^{r_{t}}(R, u)-f(R) u^{\alpha-1}$, где $f(R)$ - начальное условие. Обратное преобразование Лапласа полученного выражения по переменной $u$ приводит к обобшенному уравнению Фоккера-Планка

$$
p^{r_{t}}(R, t)=f(R)+\frac{1}{\Gamma(\alpha)} \int_{0}^{t} d \tau(t-\tau)^{\alpha-1}\left[\hat{L} p^{r_{t}}\right](R, \tau) .
$$

Это уравнение можно также представить в эквивалентной форме

$$
\frac{\partial^{\alpha} p^{r_{t}}(R, t)}{\partial t^{\alpha}}-\frac{f(R) t^{-\alpha}}{\Gamma(1-\alpha)}=\hat{L} p^{r_{t}}(R, t)
$$

через дифференциальный оператор Римана-Лиувилля $\partial^{\alpha} / \partial t^{\alpha}$ (дробная производная по времени) порядка $\alpha[1]$. Если плотность вероятности $p^{R}(R, \tau)$ известна явно (как например, в случае гармонического потенциала, который приводит к линейному полю сил в стандартном уравнении Фоккера-Планка) или в виде разложения в ряд из процедуры разделения переменных, то решение уравнения (6) можно легко получить с помощью интегрального преобразования

$$
p^{r_{t}}(R, t)=\int_{0}^{\infty} F_{\alpha}(z) p^{R}\left(R, t^{\alpha} z\right) d z
$$

В этой связи следует заметить, что вывод уравнения (6), имеющего дробный интеграл по времени, не является тривиальным, поскольку соотношение подчиненности

$$
p^{r_{t}}(R, t)=\int_{0}^{\infty} p^{R}(R, \tau) p^{S}(t, \tau) d \tau
$$

не есть свертка. Представление аномальной диффузии с помошью двух случайных процессов, связанных обшим непрерывным параметром, было предложено в [28] и затем исследовалось в [29]. Однако в этих работах процесс $S(t)$ не был определен явно.

Если теперь обратить внимание на степенное ядро интегрального уравнения (6), то становится ясно, что оно описывает долговременные эффекты памяти, характерные для подчиненного процесса. Появление такого ядра является прямым следствием свойства распределения вероятности направляюшего процесса. Индекс $\alpha$ определяет как параметр устойчивого распределения времени ожидания $T(\tau)$, так и порядок дробного интеграла (или производной) по времени в соответствуюшем обобщенном уравнении Фоккера-Планка. Как показано в [30], благодаря долговременным эффектам памяти сложное поведение микроскопической динамики таких систем может передаваться на макроскопический уровень, что проявляется в некотором упорядочении макроскопического движения.

Важно подчеркнуть, что в нашем рассмотрении индекс $\alpha$ принимает значение $0<\alpha \leqslant 1$. Формально уравнения (6) и (7) можно рассматривать и с индексом $1<\alpha \leqslant 2$ (супердиффузия). Однако в этом случае функции $p^{S}(t, \tau)$ и $p^{r t}(R, t)$ перестают быть плотностями вероятности. Более подробно этот вопрос рассмотрен в работе [31]. В то

6 Теоретическая и математическая физика, т. 138, № 3, 2004 г. 
же время если перейти к более общей модели случайных блужданий, в которой случайные времена ожидания $T_{i}$ и скачки случайной длины $R_{i}$ являются зависимыми величинами, то случайные процессы, порождаемые ими, могут описывать при определенных условиях супердиффузное поведение. Мы не будем рассматривать эту модель и ограничимся только упоминанием работы [32], где можно найти ее детальное обсуждение.

В литературе [6], [33] неоднократно отмечался тот факт, что аномальная диффузия, описываемая уравнениями (6) и (7), не требует никакой модификации гиббсовской термодинамики. Теперь становятся более понятными основания такого вывода. Применим приведенный выше подход к процессу Орнштейна-Уленбека. Как хорошо известно, движение точечной частицы со скоростью $V(\tau)$ в термостате определяется вязким трением $\gamma V$ и случайными столкновениями $W(\tau)$ в форме уравнения

$$
d V(\tau)=-\gamma V(\tau) d \tau+d W(\tau)
$$

Как обычно, $W(\tau)$ - винеровский процесс с нулевым средним и дисперсией $2 D$. Тогда дисперсия случайной величины $V$ есть

$$
\left\langle V_{i}^{2}(\tau)\right\rangle=v_{i, 0}^{2} e^{-2 \gamma \tau}+\frac{D}{\gamma}\left(1-e^{-2 \gamma \tau}\right)
$$

где $v_{i, 0}$ - начальное условие. Теперь проведем рандомизацию временной переменной $\tau$ этого процесса, заменив ее на стохастический процесс $S(t)$. Используя интегральное соотношение (4) и полагая процессы $S(t)$ и $V(\tau)$ независимыми, находим дисперсию случайной величины $V[S(t)]$. При интегрировании экспоненциальной функции с функцией $F_{\alpha}(z)$ мы получаем функцию Миттаг-Лефлера. Полагая, что начальное условие остается таким же, как для процесса Орнштейна-Уленбека, в результате находим

$$
\left\langle V_{i}^{2}[S(t)]\right\rangle=v_{i, 0}^{2} E_{\alpha}\left(-2 \gamma t^{\alpha}\right)+\frac{D}{\gamma}\left(1-E_{\alpha}\left(-2 \gamma t^{\alpha}\right)\right)
$$

Отсюда видно, что стационарное состояние для процесса $V[S(t)]$ существует и что оно конечно. Стационарные состояния для уравнений (6), (7) существуют также и в других случаях (например, для гармонического потенциала [7], [33]). Поэтому трансформация марковского процесса в подчиненный процесс, представленный выше, не нарушает закон равнораспределения энергии по степеням свободы. При наличии стационарных состояний понятие температуры сохраняет свой смысл для систем типа (6). Направляюший процесс $S(t)$ меняет только характер релаксации в таких системах, делая его медленным, степенным. В данном случае флуктуационно-диссипационная теорема и Н-теорема выполняются в той же мере, как и для процессов нормальной диффузии. Потребности в статистике Цалиса не возникает. 


\section{4. ОБОБШЕНИЕ ОСНОВНОГО КИНЕТИЧЕСКОГО УРАВНЕНИЯ}

Предложенный выше подход может быть расширен на основное кинетическое уравнение. Это уравнение представляет собой разновидность уравнения Чепмена-Колмогорова для марковских процессов. Если уравнение Чепмена-Колмогорова является интегральным, то основное кинетическое уравнение имеет дифференциальную форму. Оно проше в обрашении и тесно связано с физикой [34].

Если множество дискретных состояний некоторой марковской системы пронумеровать индексом $n$, то основное кинетическое уравнение примет вид

$$
\frac{d p_{n}(t)}{d t}=\sum_{k=0}^{\infty}\left[W_{n k} p_{k}(t)-W_{k n} p_{n}(t)\right]
$$

где $W_{k n}$ - вероятность перехода за единицу времени из состояния $n$ в состояние $k$. Уравнение (10) определяет вероятность $p_{n}$ для системы оказаться в состоянии $n$. Для непрерывного множества состояний уравнение (10) можно представить как

$$
\frac{d P(y, t)}{d t}=\int\left[W\left(y \mid y^{\prime}\right) P\left(y^{\prime}, t\right)-W\left(y^{\prime} \mid y\right) P(y, t)\right] d y^{\prime}
$$

с начальным условием $P(y, 0)$.

Для удобства дальнейших вычислений запишем оба случая (дискретньй и непрерывньй) в компактном виде

$$
\frac{d p(t)}{d t}=\widehat{\mathrm{W}} p(t),
$$

где $\widehat{\mathrm{W}}$ обозначает оператор перехода между состояниями системы. Важно подчеркнуть, что оператор $\widehat{\mathrm{W}}$ не зависит от времени. Преобразованием Лапласа

$$
\tilde{p}(s)=\int_{0}^{\infty} e^{-s t} p(t) d t
$$

уравнение (11) сводится к выражению

$$
\widehat{\mathrm{W}} \tilde{p}(s)=s \tilde{p}(s)-p(0) .
$$

Далее мы определяем новый случайный процесс

$$
p_{\alpha}(t)=\int_{0}^{\infty} p^{S}(t, \tau) p(\tau) d \tau
$$

Он представляет собой марковский процесс (подчиненный процесс), направляемый случайным процессом $S(t)$ (см. раздел 2 и приложение). В пространстве изображений функции $p_{\alpha}(t)$ и $p(t)$ связаны равенством $\tilde{p}_{\alpha}(s)=s^{\alpha-1} \tilde{p}\left(s^{\alpha}\right)$, где

$$
\tilde{p}_{\alpha}(s)=\int_{0}^{\infty} e^{-s t} p_{\alpha}(t) d t
$$


есть преобразование Лапласа функции $p_{\alpha}(t)$. Легко установить, что

$$
\begin{aligned}
\widehat{\mathrm{W}} \tilde{p}_{\alpha}(s) & =s^{\alpha-1} \widehat{\mathrm{W}} \tilde{p}\left(s^{\alpha}\right)= \\
& =s^{\alpha-1}\left[s^{\alpha} \tilde{p}\left(s^{\alpha}\right)-p(0)\right]= \\
& =s^{\alpha} \tilde{p}_{\alpha}(s)-p(0) s^{\alpha-1} .
\end{aligned}
$$

Применяя обратное преобразование Лапласа к (12), можно записать уравнение (11) в обобщенном виде

$$
p_{\alpha}(t)=p(0)+\frac{1}{\Gamma(\alpha)} \int_{0}^{t} d \tau(t-\tau)^{\alpha-1} \widehat{\mathrm{W}} p_{\alpha}(\tau)
$$

через дробный интеграл по времени. Для $\alpha=1$ мы сразу получаем выражение (11) в интегральной форме.

При анализе дискретных марковских процессов используют производящую функцию вероятности

$$
G(\zeta, t)=\sum_{k=0}^{\infty} \zeta^{k} p_{k}(t)
$$

где $\zeta$ - вспомогательная переменная, принимаюшая такие значения $|\zeta| \leqslant 1$, чтобы ряд сходился. Поскольку $\sum_{k=0}^{\infty} p_{k}(t)=1$ и $p_{k}(t) \geqslant 0$, эта функция наверняка существует при $|\zeta|=1$. С помощью производящей функции легко найти моменты такого процесса, если взять производные от производяшей функции по $\zeta$ и положить затем в них $\zeta=1$. Производящая функция процесса, направляемого стохастическим "таймером" $S(t)$, имеет вид

$$
G_{\alpha}(\zeta, t)=\int_{0}^{\infty} F_{\alpha}(z) G\left(\zeta, t^{\alpha} z\right) d z
$$

Следовательно, зная производящую функцию любого дискретного марковского процесса (см. [34], п.п. 1.1.5, 1.4.1, и [35], гл. 6), можно сразу найти производяшую функцию подчиненного процесса интегрированием выражения (14).

ПРИмеР 2. В качестве иллюстрации рассмотрим релаксацию в трехуровневой системе при отличных от нуля частотах перехода $w_{12}$ и $w_{23}$ и начальном условии $p_{1}(0)=$ $p_{2}(0)=0, p_{3}(0)=1$. Пусть $N$ - общее число объектов системы, которые находятся в одном из трех состояний. Число объектов по отдельности в состояниях $1,2,3$ обозначим соответственно $N_{1}, N_{2}$ и $N_{3}$. Допустим, что в момент времени $t=0$ под влиянием внешнего воздействия объекты в этой системе были распределены по своим состояниям следуюшим образом:

$$
\frac{N_{1}(t=0)}{N}=p_{1}(0)=\frac{N_{2}(t=0)}{N}=p_{2}(0)=0, \quad \frac{N_{3}(t=0)}{N}=p_{3}(0)=1 .
$$

После выключения воздействия система будет стремиться вернуться в свое равновесное состояние. Частоты переходов $w_{12}=w_{21}$ и $w_{23}=w_{32}$ (остальные равны нулю) определяются микроскопическими характеристиками системы (например, из гамильтониана взаимодействия и золотого правила Ферми). 
Как меняются вероятности состояний со временемГ В этом случае обобщенное кинетическое уравнение (13) можно записать в виде трех уравнений:

$$
\begin{aligned}
& p_{1}(t)=p_{1}(0)+\frac{w_{12}}{\Gamma(\alpha)} \int_{0}^{t}(t-\tau)^{\alpha-1} p_{2}(\tau) d \tau \\
& p_{2}(t)=p_{2}(0)+\frac{1}{\Gamma(\alpha)} \int_{0}^{t}(t-\tau)^{\alpha-1}\left[w_{23} p_{3}(\tau)-w_{12} p_{2}(\tau)\right] d \tau \\
& p_{3}(t)=p_{3}(0)-\frac{w_{23}}{\Gamma(\alpha)} \int_{0}^{t}(t-\tau)^{\alpha-1} p_{3}(\tau) d \tau .
\end{aligned}
$$

Из линейности этих уравнений непосредственно следует, что

$$
p_{1}(t)+p_{2}(t)+p_{3}(t)=p_{1}(0)+p_{2}(0)+p_{3}(0)=1
$$

Уравнение для $p_{3}(t)$ не зависит от двух других уравнений и может быть сразу проинтегрировано. Затем последовательно находим $p_{2}(t)$ и $p_{1}(t)$. В результате получим

$$
\begin{aligned}
& p_{1}(t)=\frac{w_{12} w_{23}}{w_{12}-w_{23}}\left[\left(E_{\alpha}\left(-w_{12} t^{\alpha}\right)-1\right) w_{12}^{-1}-\left(E_{\alpha}\left(-w_{23} t^{\alpha}\right)-1\right) w_{23}^{-1}\right], \\
& p_{2}(t)=\frac{w_{23}}{w_{12}-w_{23}}\left[E_{\alpha}\left(-w_{23} t^{\alpha}\right)-E_{\alpha}\left(-w_{12} t^{\alpha}\right)\right], \\
& p_{3}(t)=E_{\alpha}\left(-w_{23} t^{\alpha}\right) .
\end{aligned}
$$

Эти решения выражаются через функцию Миттаг-Лефлера. Как хорошо известно [36], эта функция имеет при $\alpha \neq 1$ степенную асимптотику. Поэтому сами решения проявляют степенную релаксацию при $t \rightarrow \infty$. Следовательно, рандомизация временных часов марковского процесса сушественно меняет характер релаксации, замедляя его. В этой связи следует упомянуть экспериментальные кривые релаксации, наблюдаемой в стеклах, которые показывают похожее асимптотическое поведение [37].

\section{5. ФУНКЦИЯ ПАМЯТИ В ПРОЦЕДУРЕ УСРЕДНЕНИЯ ГЛАДКОЙ ФУНКЦИИ ПО МНОЖЕСТВУ КАНТОРА}

В этом разделе мы обсудим процедуру усреднения гладкой функции по множеству Кантора и покажем, что она не имеет последовательной прямой связи с дробным интегралом. Будем следовать обозначениям книги [17]. Пусть функция памяти $K(t)$ задана на отрезке $[0, T]$. Представим эту функцию в виде обычных ступенчатых функций на этом отрезке. Их ширина определяется процедурой построения бинарного множества Кантора размерности $\nu=\ln 2 / \ln (1 / \xi)$. Высота у всех ступенчатых функций одинаковая, но удовлетворяет условию нормировки $\int_{0}^{T} K(t) d t=1$. В этом случае лаплас-образ функции памяти $K_{2}^{(N)}(t)$ на $N$-м этапе построения канторовского множества определяется выражением

$$
\bar{K}_{2}^{(N)}(p)=\int_{0}^{\infty} e^{-p t} K_{2}^{(N)}(t) d t=\frac{1-e^{-p T \xi^{N}}}{p T \xi^{N}} Q_{2}^{(N)}[p T(1-\xi)],
$$


где

$$
Q_{2}^{(N)}(z)=\prod_{n=0}^{N-1} \frac{1+e^{-z \xi^{n}}}{2}, \quad z=(1-\xi) p T .
$$

Числитель дроби в (15) стремится к 2 при $n \gg 1$. Поэтому в пределе $N \rightarrow \infty$ произведение (15) сходится к некоторой функции $Q_{2}(z)$. Эта функция удовлетворяет функциональному уравнению $Q_{2}(z)=\left(1+e^{-z}\right) Q_{2}(z \xi) / 2$, которое для $z \gg 1$ упрощается, принимая вид $Q_{2}(z) \approx Q_{2}(z \xi) / 2$.

Для случая $M$ полосок выражение лаплас-образа функции памяти приведено в книге [17] (п. 5.3.1). В этой же книге полученные результаты обобшены с регулярного множества Кантора на случай, когда параметры являются случайными (п. 5.3.2) или сами полоски имеют случайное положение (п. 5.3.4). Также в [17] рассмотрены канторовские полоски с экспоненциальным затуханием (п. 5.3.3). Все упомянутые результаты можно записать в компактной форме

$$
\bar{K}^{\Sigma}(p)=\lim _{N \rightarrow \infty} \widehat{G}^{(N)}(p)=G(z)=\prod_{n=0}^{\infty} \hat{g}\left(z \xi^{n}\right), \quad z=(1-\xi) p T
$$

Здесь $\hat{g}(x)$ - целая положительная функция, которую можно представить рядом $\ln \hat{g}(x)=\sum_{k=1}^{\infty} c_{k} x^{k} / k$ !, где $c_{k}$ - некоторые константы. Полагаем, что произведение (16) сходится. Получить представление любой приемлемой функции типа $\ln \hat{g}(x)$ в виде ряда Тейлора не представляет особого труда с помощью компьютерных программ аналитических вычислений. Следует заметить, что произведение (16) будет тоже целой функцией, которая не имеет нулей на всей конечной комплексной плоскости, а значит, может быть представлена в виде $G(z)=e^{A(z)}$, где $A(z)$ - целая функция [38]. Функция $G(z)$ имеет сушественную сингулярную точку на бесконечности и стремится к нулю при $\operatorname{Re} z \rightarrow \infty$. Это указывает на неаналитическое поведение асимптотики этой функции на бесконечности.

Представляет интерес найти вид самой функции $A(z)$. Логарифмирование произведения $\bar{K}^{\Sigma}(p)$ преобразовывает его в сходящийся ряд. При этом искомая функция записывается как

$$
A(z)=\sum_{n=0}^{\infty} \ln \hat{g}\left(z \xi^{n}\right)=\sum_{n=0}^{\infty} h(n)
$$

где $z$ играет роль параметра. С помошью формулы суммирования Пуассона

$$
\sum_{n=0}^{\infty} h(n)=\frac{1}{2} h(0)+\int_{0}^{\infty} h(x) d x+2 \sum_{m=1}^{\infty} \int_{0}^{\infty} h(x) \cos (2 \pi m x) d x
$$

где $h(x)=\ln \hat{g}\left[e^{x \ln \xi} z\right]$, и замены переменных $y=e^{x \ln \xi}$ находим

$$
A(z)=\frac{1}{2} \ln \hat{g}(z)+\frac{1}{\ln \xi} \sum_{m=-\infty}^{\infty} \int_{1}^{\infty} y^{-1+2 \pi m i / \ln \xi} \ln \hat{g}(y z) d y
$$


В выражении

$$
\frac{1}{\ln \xi} \sum_{m=-\infty}^{\infty} \int_{0}^{\infty} y^{-1+2 \pi m i / \ln \xi} \ln \hat{g}(y z) d y
$$

интеграл можно представить в виде суммы двух интегралов с интегрированием в пределах от 0 до 1 и от 1 до $\infty$. Кроме того, согласно [20] это выражение в точности равно нулю, а поэтому возможен переход от интеграла с пределами от 1 до $\infty$ в (17) к интегралу от 0 до 1 ,

$$
A(z)=\frac{1}{2} \ln \hat{g}(z)-\frac{1}{\ln \xi} \sum_{m=-\infty}^{\infty} \int_{0}^{1} y^{-1+2 \pi m i / \ln \xi} \ln \hat{g}(y z) d y .
$$

Теперь разложим функцию $\ln \hat{g}(y z)$ в ряд Тейлора и проинтегрируем (18) по $y$. В результате получим

$$
A(z)=\frac{1}{2} \ln \hat{g}(z)-\frac{1}{\ln \xi} \sum_{m=-\infty}^{\infty} \sum_{k=1}^{\infty} \frac{c_{k} z^{k}}{k !(k+2 \pi m i / \ln \xi)}
$$

Используя тождество [39]

$$
\sum_{m=-\infty}^{\infty} \frac{1}{k+2 \pi m i / \ln \xi}=\frac{1}{2} \ln \xi \operatorname{cth}\left(\frac{k}{2} \ln \xi\right)
$$

и меняя порядок суммирования в двойной сумме (19) в силу ее сходимости, после простых арифметических преобразований получаем

$$
A(z)=\sum_{k=1}^{\infty} \frac{c_{k} z^{k}}{k !\left(1-\xi^{k}\right)}
$$

Таким образом, мы находим экспоненциальное представление лаплас-образа функции памяти $\bar{K}^{\Sigma}(t)$. Прямой подстановкой нетрудно убедиться, что

$$
A(\xi z)=\sum_{k=1}^{\infty} c_{k} \frac{\xi^{k} z^{k}}{k !\left(1-\xi^{k}\right)}=\sum_{k=1}^{\infty} c_{k} \frac{z^{k}}{k !}\left(\frac{1}{1-\xi^{k}}-1\right)=A(z)-\ln \hat{g}(z) .
$$

Следовательно, выполняется также функциональное уравнение $G(\xi z)=G(z) / \hat{g}(z)$. Отсюда видно, что разложение в ряд функции $\ln \hat{g}(x)$ полностью определяет в известной степени экспоненциальную форму $\bar{K}^{\Sigma}(p)$. Если $\ln \hat{g}(z)$ - целая функция порядка $\beta=$ $\lim _{k \rightarrow \infty} \sqrt[k]{\left|c_{k}\right|}$, то $A(z)$ будет целой функцией того же самого порядка [38]. В этой связи важно подчеркнуть, что функция $\bar{K}^{\Sigma}(p)$ не является степенной, а значит, она не может точно аппроксимировать никакую степенную функцию для всех $p$. Поскольку преобразование Лапласа является однозначным отображением, то функция памяти $K^{\Sigma}(t)$, найденная согласно дискретной процедуре построения множества Кантора или полученная 
похожим образом, не приводится в точности к степенному ядру, характерному для дробного интеграла (производной). Поэтому такой подход не может быть использован для последовательной интерпретации дробного оператора.

Предел $\lim _{x \rightarrow \infty} \hat{g}(x)=\bar{g}=$ const $<1$. В частности, для бинарного множества Кантора этот предел будет равен $\lim _{x \rightarrow \infty}\left(1+e^{-x}\right) / 2=1 / 2$, а в более обшем случае множества Кантора с $M$ полосками

$$
\lim _{x \rightarrow \infty} \frac{1}{M} \frac{1-\exp \left(-\frac{x M}{M-1}\right)}{1-\exp \left(-\frac{x}{M-1}\right)}=\frac{1}{M} .
$$

Поэтому функциональное уравнение $G(\xi z)=G(z) / \hat{g}(z)$ сводится к соотношению подобного преобразования $G(\xi z) \approx G(z) / \bar{g}$. Это соотношение имеет единственное нетривиальное решение $B z^{\mu} L(z)$, где $B$ - некоторая константа, а $\mu=\ln (1 / \bar{g}) / \ln (1 / \xi), L(\xi z)=$ $L(z)$ - лог-периодическая функция. Более точное асимптотическое представление функции $G(z)$ можно найти с помошью формулы Эйлера-Маклорена [17]. Хотя в ряде случаев для $z>1$ такой асимптотический вид (после усреднения лог-периодического члена) достаточно близок к степенной функции с нецелым показателем, все же точные аналитические свойства функции $\bar{K}^{\Sigma}(p)$ полностью игнорировать нельзя.

\section{6. ВЫВОДЫ}

На основании вьшеизложенного можно сделать следуюшие выводы. Появление дробной производной (или интеграла) по времени в кинетических уравнениях отражает тот факт, что они описывают подчиненные случайные процессы. Их направляющий процесс непосредственно связан со случайным процессом, имеющим устойчивое распределение вероятности. Это своего рода стохастическая стрела времени. В отличие от традиционной, детерминированной стрелы времени с "таймером", отсчитываюшим равные интервалы времени, стохастический “таймер” имеет неравномерный шаг. Этот шаг является случайной величиной с устойчивым распределением вероятности. Такой характер распределения вероятности порождает долговременные эффекты памяти у подчиненного процесса, а релаксация в подобной системе имеет степенной спад. Хотя упомянутая трансформация стохастических процессов не нарушает законы классической термодинамики, она требует определенной модификации их макроскопического описания. Это нашло свое отражение в появлении обобшенного (дробного) оператора по времени в кинетическом описании такого рода аномальных систем. Порядок этого оператора позволяет найти параметр $\alpha$ соответствуюшего устойчивого распределения. $\mathrm{K}$ сожалению, следует констатировать, что попытки интерпретации дробных интеграла и производной в терминах фрактальной геометрии не увенчались успехом.

\section{ПРИЛОЖЕНИЕ}

Преобразование Лапласа от плотности вероятности $p^{S}(t, x)$ по переменной $x$ записывается как

$$
\bar{p}^{S}(t, v)=\int_{0}^{\infty} e^{-v x} p^{S}(t, x) d x=\left\langle e^{-v S(t)}\right\rangle=E_{\alpha}\left(-v t^{\alpha}\right) .
$$


Изображение Лапласа функции Миттаг-Лефлера $E_{\alpha}\left(-v t^{\alpha}\right)$ по переменной $t$ хорошо известно и равно $u^{\alpha-1} /\left(u^{\alpha}+v\right)[2]$. Обратное преобразование этого изображения по переменной $v$ имеет вид

$$
\hat{p}^{S}(u, x)=\int_{0}^{\infty} e^{-u t} p^{S}(t, x) d t=u^{\alpha-1} e^{-u^{\alpha} x} .
$$

Согласно формуле обрашения преобразования Лапласа для величины $\hat{p}^{S}(u, x)$ получим

$$
p^{S}(t, x)=\frac{1}{2 \pi i} \int_{\mathrm{Br}} e^{u t-x u^{\alpha}} u^{\alpha-1} d u,
$$

где $\mathrm{Br}$ обозначает контур интегрирования Бромвича. После замены переменных $u t \rightarrow u$ и $z=x / t^{\alpha}$ функцию $p^{S}(t, x)$ можно привести к виду $t^{-\alpha} F_{\alpha}(z)$, где

$$
F_{\alpha}(z)=\frac{1}{2 \pi i} \int_{\mathrm{Br}} e^{u-z u^{\alpha}} u^{\alpha-1} d u .
$$

Преобразование Меллина от функции $F_{\alpha}(z)$ есть

$$
\int_{0}^{\infty} F_{\alpha}(z) z^{s-1} d z=\frac{\Gamma(s)}{2 \pi i} \int_{\mathrm{Br}} e^{u} u^{\alpha(-s+1)-1} d u .
$$

Далее мы деформируем контур Бромвича в контур Ханкеля (На). Он начинается с точки $u=-\infty-i a(a>0)$, идет вдоль разреза по отрицательной оси действительных чисел и, огибая ноль, приходит в точку $u=-\infty+i b(b>0)$. Теперь интеграл

$$
\int_{\mathrm{Ha}} e^{u} u^{\alpha(-s+1)-1} d u=\frac{1}{\Gamma(1-\alpha+\alpha s)}
$$

становится табличным (представление Ханкеля для обратной величины от гамма-функции). Функция с меллин-образом $\Gamma(s) / \Gamma(1-\alpha+\alpha s)$ хорошо известна и принадлежит классу Н-функций [40]. В результате можно записать

$$
F_{\alpha}(z)=H_{11}^{10}\left(z \mid \begin{array}{c}
(1-\alpha, \alpha) \\
(0,1)
\end{array}\right)=\sum_{k=0}^{\infty} \frac{(-z)^{k}}{k ! \Gamma(1-\alpha-k \alpha)}
$$

Поскольку этот ряд имеет бесконечный радиус сходимости для $0<\alpha<1$, функция $F_{\alpha}(z)$ будет целой по переменной $z$. Изображение Лапласа функции $F_{\alpha}(z)$ выражается через функцию Миттаг-Лефлера,

$$
\int_{0}^{\infty} e^{-z \zeta} F_{\alpha}(z) d z=E_{\alpha}(-\zeta)
$$

Как хорошо известно [36], функция $E_{\alpha}(-\zeta)$ не только полностью монотонная для $\zeta \geqslant 0$ при $0<\alpha \leqslant 1$, но и целая, имеющая порядок $1 / \alpha$. Поэтому, согласно [26] функция $F_{\alpha}(z)$ неотрицательна для всех $z>0$. Принимая во внимание условие нормировки

$$
\int_{0}^{\infty} F_{\alpha}(z) d z=1
$$


убеждаемся, что выражение $p^{S}(t, x)$ действительно обладает свойствами плотности вероятности.

Функция $F_{\alpha}(z)$ имеет также другие интересные свойства, которые стоит кратко обсудить. Во-первых, при $\alpha=1$ она соответствует $\delta$-функции Дирака, $F_{1}(z)=\delta(z-1)$. В частных случаях $\alpha=1 / 2$ и $\alpha=1 / 3$ она выражается через хорошо известные трансцендентные функции, $F_{1 / 2}(z)=\pi^{-1 / 2} e^{-z^{2} / 4}$ и $F_{1 / 3}(z)=3^{2 / 3} A i\left(z / 3^{1 / 3}\right)$, соответственно, где $A i$ обозначает функцию Эйри [36]. При $0<\alpha \leqslant 1 / 2$ эта функция монотонно убывает. При $1 / 2<\alpha<1$ она имеет максимум в некоторой точке $z_{\max }$, зависящей от величины параметра $\alpha$. Следует подчеркнуть, что решение задач Коши для распространения сигнала в случае одномерного диффузионно-волнового уравнения с дробной производной по времени выражается именно через функцию $F_{\alpha}(z)[27]$, [30].

Благодарности. Автор выражает глубокую признательность К. Верон (K. Weron, Institute of Physics, Wroclaw University of Technology) и В. Мельнику (Радиоастрономический институт НАНУ) за многочисленные полезные обсуждения, стимулировавшие написание данной работы, а также Р.Р. Нигматуллину (Казанский университет) за возможность ознакомления с главой 5 книги [17]. Автор хочет отдельно поблагодарить анонимного рецензента за его замечания и советы.

\section{Список литературы}

[1] С. Г. Самко, А. А. Килбас, О.И. Маричев. Интегралы и производные дробного порядка и некоторые их приложения. Минск: Наука и техника, 1987.

[2] K.S. Miller, B. Ross. An Introduction to the Fractional Calculus and Fractional Differential Equations. New York: Wiley, 1993.

[3] Р.Р. Нигматуллин, Я. Е. Рябов. ФТТ. 1997. Т. 39. № 1. С. 101.

[4] K. Weron, A. Klauser. Ferroelectrics. 2000. V. 236. P. 59.

[5] M.F. Shlesinger, G. M. Zaslavsky, J. Klafter. Nature. 1993. V. 363. P. 31.

[6] R. Metzler, E. Barkai, J. Klafter. Phys. Rev. Lett. 1999. V. 82. P. 3563.

[7] R. Metzler, J. Klafter. Phys. Rep. 2000. V. 339. P. 1.

[8] G. M. Zaslavsky. Physica D. 1994. V. 76. P. 110.

[9] A.I. Saichev, G. M. Zaslavsky. Chaos. 1997. V. 7. P. 753.

[10] B. Ross (ed.). Fractional Calculus and Its Applications. Lect. Notes in Math. V. 457. New York: Springer, 1975.

[11] A. McBride, G. Roach (eds.). Fractional Calculus. Research Notes in Math. V. 138. BostonLondon-Melbourne: Pitman, 1985.

[12] K. Nishimoto (ed.). Fractional Calculus and Its Applications. Koriyama: Hihon University, 1990.

[13] P. Rusev, I. Dimovski, V. Kiryakova (eds.). Transform Methods and Special Functions. Sofia: Institute of Mathematics and Informatics, Bulgarian Academy of Sciences, 1998.

[14] B. Mandelbrot. Fractal Geometry of Nature. San-Francisco: Freeman, 1983.

[15] Л. Пьетронеро, Э. Тозатти (ред.). Фракталы в физике. Труды VI Межд. симпозиума по фракталам в физике (Триест, Италия, 9-12 июля 1985 г.). М.: Мир, 1988.

[16] Р. Р. Нигматуллин. ТМФ. 1992. Т. 90. № 3. С. 354.

[17] A. Le Mehaute, R.R. Nigmatullin, L. Nivanen. Fleches du Temps et Geometric Fractale. Paris: Hermes, 1998.

[18] А. И. Олемской, А. Я. Флат. УФН. 1993. Т. 163. № 12. С. 1; Sh. Fu. Real Anal. Exchange. 1995. V. 21(1). P. 308; F. Y. Ren, Z. G. Yu, F. Su. Phys. Lett. A. 1996. V. 219. P. 59; Z. G. Yu, F. Y. Ren. J. Phys. A. 1997. V. 30. P. 5559; F. Y. Ren et al.. Physica A. 1997. V. 246. 
P. 419; F. Y. Ren, Z. G. Yu. Progr. Natur. Sci. 1997. V. 7. P. 422; M. Monsrefi-Torbati, J. K. Hammond. J. Franklin Inst. B. 1998. V. 335. № 6. P. 1077; F. Y. Ren, J. R. Liang, X. T. Wang. Phys. Lett. A. 1999. V. 252. P. 141; Z. G. Yu. Phys. Lett. A. 1999. V. 257. P. 221; A. I. Olemskoi, V. F. Klepikov. Phys. Rep. 2000. V. 338. P. 571.

[19] Р. С. Рутман. ТМФ. 1994. Т. 100. № 3. С. 476; 1995. Т. 105. № 3. С. 393.

[20] A. A. Stanislavsky, K. Weron. Physica A. 2002. V. 303. № 1-2. P. 57.

[21] E. W. Montroll, G. H. Weiss. J. Math. Phys. 1965. V. 6. P. 167.

[22] H. Scher, E. W. Montroll. Phys. Rev. B. 1975. V. 12. P. 245; M. F. Shlesinger, B. D. Hughes. Physica A. 1981. V. 109. P. 597; M.F. Shlesinger. J. Stat. Phys. 1984. V. 36. P. 639; H. Weissman, G.H. Weiss, S. Havlin. J. Stat. Phys. 1989. V. 57. P. 301; J. Klafter, G. Zumofen. J. Phys. Chem. 1994. V. 98. P. 7366.

[23] В. М. Золотарев. Одномерные устойчивые распределения. М.: Наука, 1983.

[24] N. H. Bingham. Z. Wahrscheinlichkeitstheorie verw. Geb. 1971. V. 17. P. 1.

[25] M. M. Meerschaert, H. Scheffler. Limit theorems for continuous time random walks, 2001 (http://unr.edu/homepage/mcubed/LimitCTRW.pdf).

[26] В. Феллер. Введение в теорию вероятностей и ее приложения. М.: Мир, 1984.

[27] F. Mainardi. Chaos, Solitons and Fractals. 1996. V. 7. P. 1461.

[28] H. C. Fogedby. Phys. Rev. E. 1994. V. 50. P. 1657.

[29] H. C. Fogedby. Phys. Rev. E. 1998. V. 58. P. 1690.

[30] A. A. Stanislavsky. Phys. Rev. E. 2000. V. 61. P. 4752.

[31] I. M. Sokolov. Phys. Rev. E. 2001. V. 63. P. 056111.

[32] P. Becker-Kern, M. M. Meerschaert, H. Scheffler. Limit theorems for coupled continuous time random walks, 2001 (http://unr.edu/homepage/mcubed/CoupleCTRW.pdf).

[33] E. Barkai, R. J. Silbey. J. Phys. Chem. B. 2000. V. 104. P. 3866; I. M. Sokolov, J. Klafter, A. Blumen. Phys. Rev. E. 2001. V. 64. P. 021107.

[34] Н. Г. ван Кампен. Стохастические процессы в физике и химил. М.: Высшая школа, 1990.

[35] Г. Рёпке. Неравновесная статистическая механика. М.: Мир, 1990.

[36] Г. Бейтмен, А. Эрдейи. Высшие трансцендентные функции. Т. 3. М.: Наука, 1967.

[37] W. G. Glöckle, T. F. Nonnenmacher. Macromolecules. 1991. V. 24. P. 6426.

[38] М. А. Лаврентьев, Б. В. Шабат. Методы теории функций комплексного переменного. М.: Наука, 1987.

[39] И. С. Градитейн, И. М. Рыжик. Таблицы интегралов, сумм, рядов и произведений. М.: Наука, 1971.

[40] C. Fox. Trans. Am. Math. Soc. 1961. V. 98. P. 395.

Поступила в редакцию 4.II.2003 г., после доработки 20.V.2003 г. 